\title{
HNF-6-independent differentiation of mouse embryonic stem cells into insulin-producing cells
}

\author{
N. Houard, G. G. Rousseau, F. P. Lemaigre \\ HORM Université Catholique de Lourain and Institute of Cellular Pathology, Brussels, Belgium
}

\begin{abstract}
Aims/hypothesis. Embryonic stem cells, when grown as embryoid bodies, spontaneously generate insulinproducing cells which could be used in therapy of diabetes mellitus, provided that their selection and differentiation are optimized. To achieve such optimization, one needs to know whether the differentiation of cells in embryoid bodies mimicks that of pancreatic beta cells in embryos. To address this question we verified if the differentiation of the insulin-producing cells in embryoid bodies requires Hepatocyte Nuclear Factor-6 (HNF-6), a transcription factor known to control pancreatic endocrine differentiation in embryos.

Methods. We generated mouse Hnf6-/- embryonic stem cells and grew them as embryoid bodies. The expression of HNF-6, insulin, and transcription factors that are regulated by HNF-6 in developing pancreas was compared in wild-type and Hnf6-/- embryoid bodies.
\end{abstract}

Results. No difference was observed in the expression of insulin between wild-type and Hnf6-/- embryoid bodies. In both cases insulin was expressed in the outer layer of cells, which is similar to the visceral endoderm. In wild-type embryoid bodies HNF-6 was transiently expressed in the outer layer of cells, but was not co-expressed with insulin. The expression of genes that are targets of HNF-6 in developing pancreas was unaffected in Hnf6-/- embryoid bodies.

Conclusion/interpretation. In contrast to the development of pancreatic beta cells, the differentiation of insulin-producing cells in embryoid bodies did not require HNF-6. Thus, the differentiation mechanism of insulin-producing cells in embryoid bodies differs from that of the beta cells and it is likely to resemble that of insulin-producing cells in the visceral endoderm. [Diabetologia (2003) 46:378-385]

Keywords HNF-6, embryonic stem cells, embryoid bodies, beta cells, cell therapy, diabetes mellitus, visceral endoderm, insulin.
Received: 30 July 2002 / Revised: 13 September 2002 Published online: 27 February 2003

(C) Springer-Verlag 2003

Corresponding author: F. P. Lemaigre, HORM Université Catholique de Lourain and Institute of Cellular Pathology, 75 Avenue Hippocrate, box 7529, 1200 Brussels, Belgium E-mail: lemaigre@horm.ucl.ac.be

Abbreviations: HNF, Hepatocyte Nuclear Factor; TBP, TATA box-binding protein; NGN-3, neurogenin-3; PDX-1, Pancreatic duodenal homeobox transcription factor-1; $\mathrm{EB}$, embryoid body; ES cell, embryonic stem cell; LIF, Leukemia Inhibitory Factor; RT-PCR, reverse transcription-polymerase chain reaction.
The low availability of pancreatic islets from donors to treat diabetes mellitus by transplantation has stimulated research to engineer insulin-producing beta cells in culture. Genetically modified lines of tumour cells or immortalized cells represent potential tools but their use suffers from a number of limitations, such as tumorigenicity, genetic instability or inappropriate differentiation $[1,2]$. Pluripotent adult or embryonic stem cells that can be differentiated into insulin-producing cells have therefore attracted much attention. Cells isolated from pancreatic ducts or from the gastrointestinal tract could be differentiated or genetically manipulated to produce insulin $[3,4,5]$ and were capable of reversing insulin-dependent diabetes in mice 
$[3,5]$. Similarly, mouse embryonic stem (ES) cells secreting insulin were selected using a gene-trap strategy in vitro and could normalize glycaemia in diabetic mice, but this effect was only transient in $40 \%$ of the mice [6]. Another method based on the selection of nestin-positive precursor cells allowed to differentiate mouse ES cells in vitro into insulin-producing cells, which, when injected in animals, organized into vascularized clusters [7]. However, these cells contained about 50 times less insulin than normal beta cells and were unable to normalize the glycaemia of diabetic mice. More recently, human ES cells were also shown to spontaneously differentiate into insulin-producing cells when grown as aggregates, showing the potential usefulness of human ES cells to treat diabetes [8]. Still, these cells did not seem to secrete insulin in response to glucose stimulation. Despite these encouraging results more information is needed to establish the culture conditions that would enable one to obtain cells that phenotypically match the pancreatic beta cells. Ideally, one should be able to reproduce in culture the biological events that govern differentiation of beta cells in the embryo [9].

ES cells can be grown in suspension as aggregates that resemble the normal embryos at the egg cylinder stage and are therefore called embryoid bodies (EBs). These aggregates mimic embryonic development and spontaneously form cell types derived from the three germ layers, for instance, cardiomyocytes, skeletal myocytes, endothelial cells, haematopoietic cells, visceral endoderm cells, neurons $[10,11,12]$. The procedure used in other studies $[6,8]$ to differentiate ES cells into insulin-producing cells started with the generation of EBs in which a small percentage of insulinproducing cells differentiate spontaneously. Work by developmental biologists identified signalling molecules and transcription factors that are required for the differentiation of pancreatic endocrine cells during embryonic development $[13,14]$. The knowledge of these signalling molecules and transcription factors could ideally be applied to EBs to improve their differentiation into insulin-producing cells [9]. However, this approach would be valid only if the differentiation of the insulin-producing cells in EBs mimics closely enough the differentiation of the beta cells in embryos.

In our study we verify if differentiation of the insulin-producing cells in EBs has the same transcription factor requirements as the pancreatic beta cells in the embryo. To do this, we tested if the differentiation of ES cells into insulin-producing cells requires the transcription factor Hepatocyte Nuclear Factor (HNF)-6. This factor belongs to the Onecut class of homeoproteins $[15,16]$ and is crucial for pancreatic endocrine cell differentiation. During pancreas development, HNF-6 is expressed in the pre-pancreatic endoderm and in the pluripotent pancreatic epithelium that gives rise to the endocrine islets, to the exocrine acini and to the ducts. It is also weakly expressed in the acinar and ductal cells but not in the differentiated endocrine cells [17, 18, 19]. Gene knock-out studies in mice have shown that in the absence of HNF-6 the number of endocrine cells at birth is dramatically reduced [19]. This resulted from the absence of activation by HNF-6 of the gene coding for neurogenin-3 (NGN-3), a transcription factor that is necessary for differentiation of pancreatic endocrine precursors [20, 21, 22]. To test the role of HNF-6 in ES cell differentiation, we generated homozygous null Hnf6-/- ES cells and compared the production of insulin by wild-type and Hnf6-/-EBs.

\section{Materials and methods}

Culture of embryonic stem cells and embryoid bodies. The ES cell line R1 was routinely cultured on a primary embryonic fibroblast feeder layer [23]. ES cell medium was Glasgow Minimum Essential Media (BHK-21) (GIBCO, Merelbeke, Belgium) containing $10 \%$ foetal bovine serum (HyClone, Verviers, Belgium), $2 \mathrm{mmol} / \mathrm{l}$ L-glutamine, $100 \mathrm{U} / \mathrm{ml}$ penicillin, $100 \mu \mathrm{g} / \mathrm{ml}$ streptomycin, $1 \%$ non-essential amino acids, $1 \%$ sodium pyruvate, $0.1 \mathrm{mmol} / 1$ 2-mercaptoethanol, supplemented with 1000 units/ml Leukemia Inhibitory Factor (LIF; Biognost, Wevelgem, Belgium). ES cells were induced to differentiate to form EBs [23]. ES cells $\left(3 \times 10^{6}\right)$ were plated on gelatin-coated tissue culture dishes (Nunc, Leuven, Belgium) and grown for 3 days in the absence of feeder fibroblasts and LIF. After addition of trypsin and EDTA, ES cell clumps were collected, seeded on bacterial grade Petri dishes containing ES cell medium supplemented with $10 \%$ foetal bovine serum, and grown in suspension for the specified duration. Day 1 of differentiation corresponded to $24 \mathrm{~h}$ of culture in bacterial grade Petri dishes. Nicotinamide (Sigma, Bornem, Belgium), a stimulator of endocrine differentiation [6, 7, 24], was added at $10 \mathrm{mmol} / \mathrm{l}$ from day 8 of differentiation onwards, as indicated in the figures.

Selection of Hnf6-/- embryonic stem cells. R1 ES cells homozygous for a targeted mutation in the HNF-6 gene (Hnf6-/-) were generated [25]. Hnf6+/- ES cells [19] were plated $\left(5 \times 10^{6}\right.$ cells per dish) on a feeder layer of neomycin-resistant embryonic fibroblasts in ES cell medium supplemented with $10 \%$ foetal bovine serum, 1000 units/ml LIF and $3 \mathrm{mg} / \mathrm{ml} \mathrm{G} 418$. After 9 days in culture the surviving colonies were expanded and their genotype was verified by PCR and Southern blotting [19].

RNA purification and reverse transcription-polymerase chain reaction $(R T-P C R)$. Total RNA was isolated from ES cells or EBs with the High Pure RNA Isolation kit (Roche, Brussels, Belgium). Total RNA was reverse transcribed for $1 \mathrm{~h}$ at $37^{\circ} \mathrm{C}$ with random primers and with M-MLV reverse transcriptase (Gibco, Merelbeke, Belgium). Semi-quantitative PCR was carried out with the following primers: 5'-AGCCCTGGAGCAAACTCAAGTCG-3' and 5'-TGCATGTAGAGTTCGACGTTGGAC-3' for Hnf6 (PCR product of 202 bp for $H n f 6 \alpha$ and of 280 bp for $H n f 6 \beta$ ), 5'-CTCGCTGGGATCACTGGAG-3' and 5'-CGGTCAAGTTCAACATCAC-3' for Pancreatic duodenal homeobox transcription factor-1 $(P d x 1$; PCR product of 406 bp), 5'-TGGCGCCTCATCCCTTGGATG-3' and 5'-CAGTCACCCACTTCTGCTTCG-3' for Ngn3 (PCR product of 

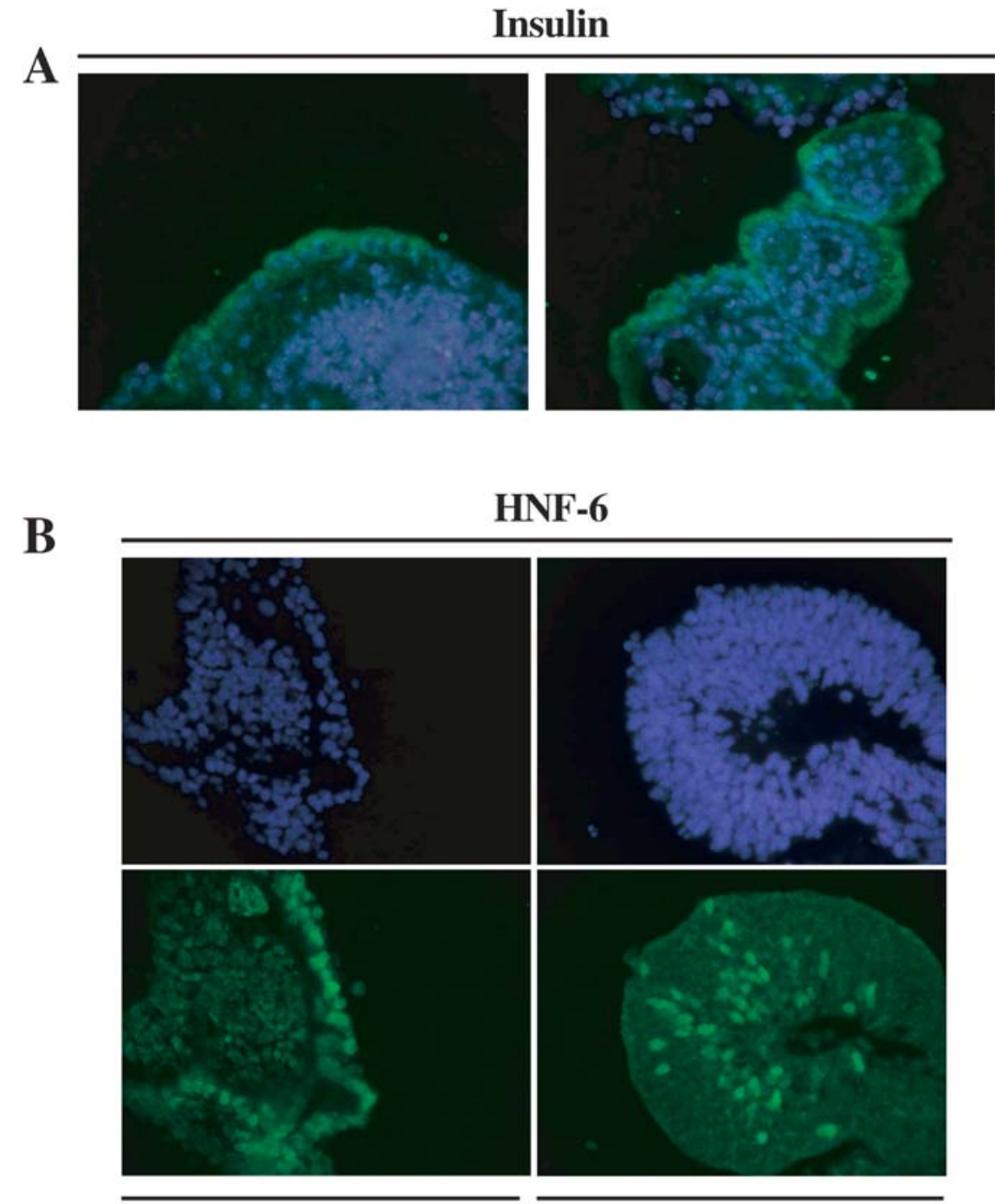

\section{Day 7}

Fig. 1A, B. Expression of insulin and of HNF-6 in differentiating embryoid bodies. A Immunofluorescence. Sections of EBs at day 21 of differentiation were subjected to immunofluorescence with DAPI to stain the nuclei (blue), and with an anti-insulin antibody (green). Insulin staining was cytoplasmic. The DAPI and antibody stainings were detected independently and the images were fused (original magnifications: left panel x40, right panel x20). B Immunofluorescence. Sections of EBs at days 7 and 21 of differentiation were stained with DAPI to visualize the nuclei (blue staining; upper panels) or with an antiHNF-6 antibody (green staining; lower panels). HNF-6 staining was nuclear. The original magnification was $\mathrm{x} 40$

160 bp), 5'-CCACCAGCCCTAAGTGATCC-3' and 5'-AAGGTCTGAAGGTCACCTGC-3' for insulinII ( PCR product of $283 \mathrm{bp}$ ), 5'-GAAAGCAACGGGAGATCCTC-3' and 5'CCTCCACTAAGGCCTCCCTC- $3^{\prime}$ for $H n f l b$ (PCR product of 279 bp), 5'-ACCCTTCACCAATGACTCCTATG-3' and 5' ATGATGACTGCAGCAAATCGC- $3^{\prime}$ for the TATA box-binding protein ( $\mathrm{Tbp}$; PCR product of $190 \mathrm{bp}$ ), 5'-CGGAATGGTCGCCTCATTC-3' and 5'-CAGTCCTGATACACTTCGTC-3' for Glut2 (PCR product of $162 \mathrm{bp}$ ). To avoid amplification of
HNF-6

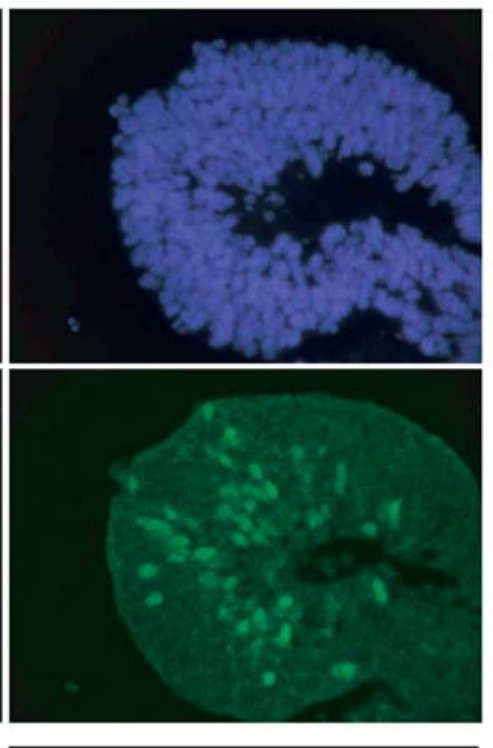

Day 21

contaminants, RT-PCR was carried out in control reactions without RNA or in the absence of reverse transcriptase.

Immunofluorescence. Wild-type or Hnf6-/- EBs were fixed in Bouin's solution or in $4 \%$ paraformaldehyde in phosphate-buffered saline (PBS), embedded in paraffin, sectioned at $5 \mu \mathrm{m}$ and immunostained. Mouse monoclonal anti-insulin (1:200; Sigma, Bornem, Belgium) and rabbit polyclonal anti-HNF-6 (1:50) antibodies were detected by immunofluorescence using biotinylated sheep anti-rabbit or anti-mouse immunoglobulin G (Roche, Brussels, Belgium) followed by streptavidin-Alexa Fluor 488 conjugate (Molecular Probes, Netherlands). The EBs analyzed by immunofluorescence were nicotinamide-treated, except for the detection of HNF-6 at day 7 of EB differentiation.

\section{Results}

Expression of insulin in mouse embryoid bodies. To validate our mouse ES cell line R1 as a source of insulin-producing cells we first examined if these cells expressed insulin upon differentiating into EBs. The ES cells were grown in suspension in the absence of LIF 

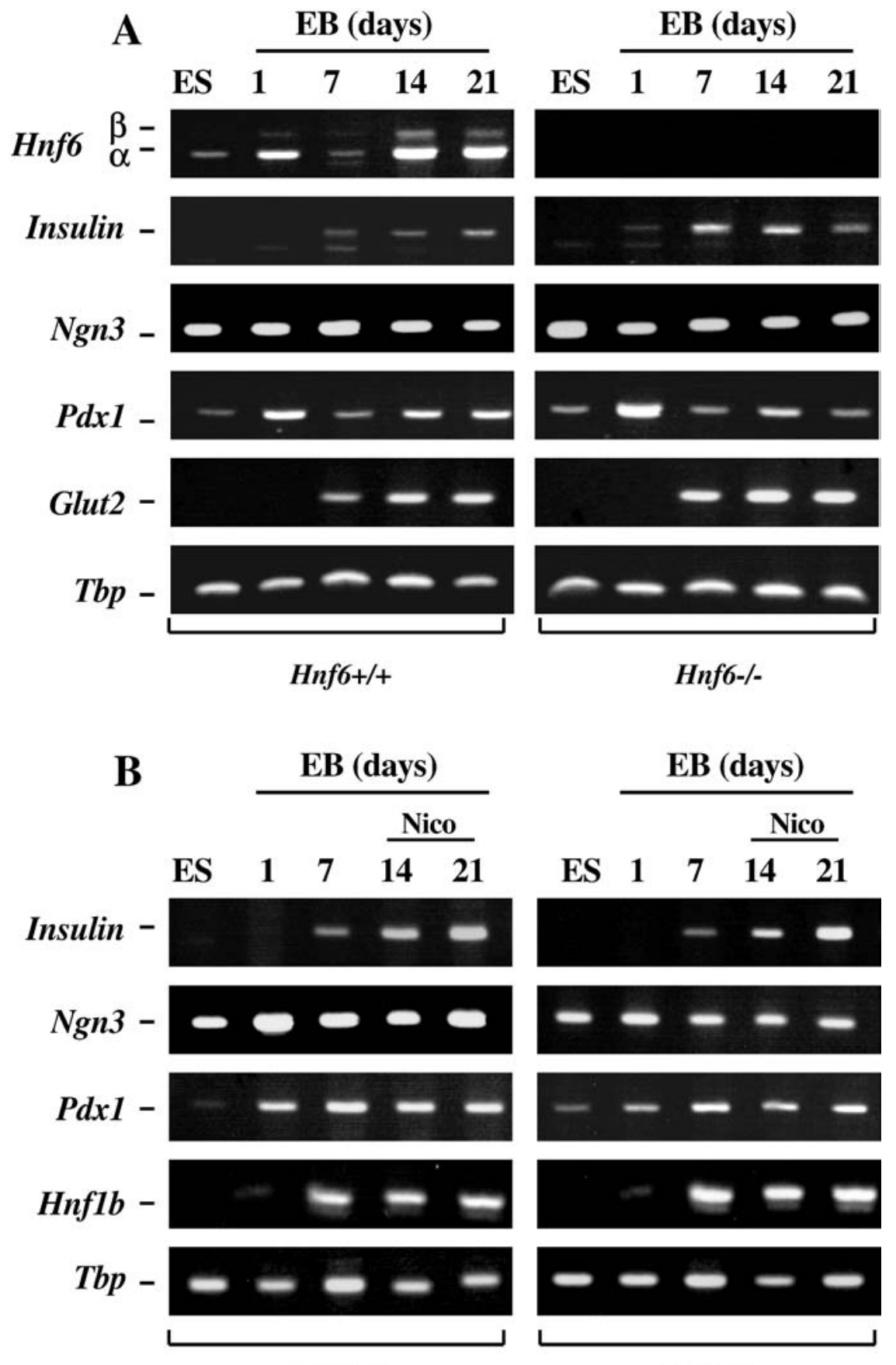

\section{Hnf6+/+}

Fig. 2A, B. Expression of genes in wild-type and Hnf6-/- embryoid bodies. Wild-type (Hnf6+/+) or Hnf6-/- undifferentiated ES cells (ES) or EBs were analyzed by RT-PCR for expression of the genes indicated. The EBs were collected at various days of differentiation as indicated above the lanes. In panel $\mathbf{B}$, the EBs were treated with $10 \mathrm{mmol} / \mathrm{l}$ nicotinamide (Nico) from day 8 till day 21 of differentiation. $T b p$ was used as a control for RNA preparation and amplification

to allow formation and differentiation of the EBs. The EBs were analyzed by immunofluorescence after 21 days of suspension culture, a period of time after which insulin should be expressed. The culture was heterogenous and contained EBs of various sizes. We detected insulin in only a subset of EBs, and in all these cases, insulin was found exclusively in the outer cell layer of the EBs (Fig. 1A). In some EBs nearly all peripheral cells expressed insulin (Fig. 1A, right panel).

To assess the time-course of the expression of insulin, RNA was extracted at various time points of the EB culture for analysis by RT-PCR (Fig. 2A). Insulin mRNA was expressed starting at day 7 of EB differentiation and its concentration remained stable until day 21 . Since the insulin mRNA content was low, we incubated the EBs with $10 \mathrm{mmol} / \mathrm{l}$ nicotinamide, a known stimulator of endocrine differentiation. Nicotinamide was added to the EB culture starting on day 8 
A
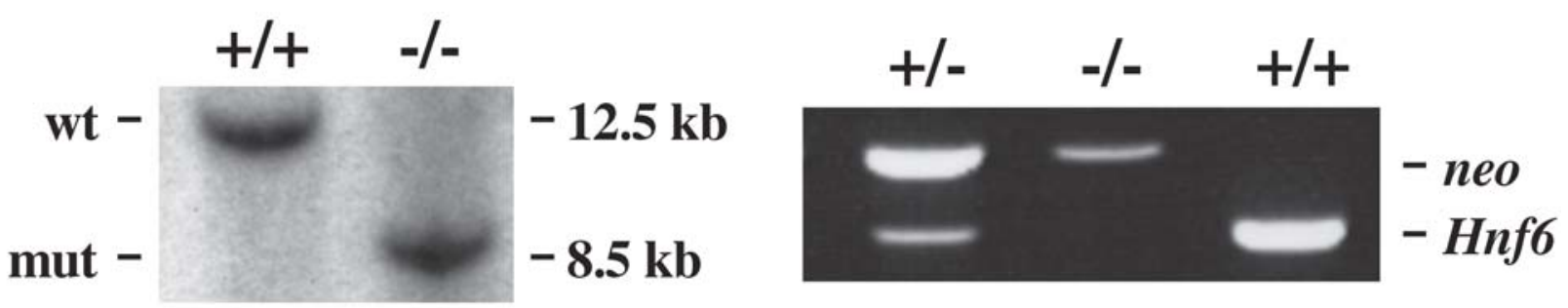

B
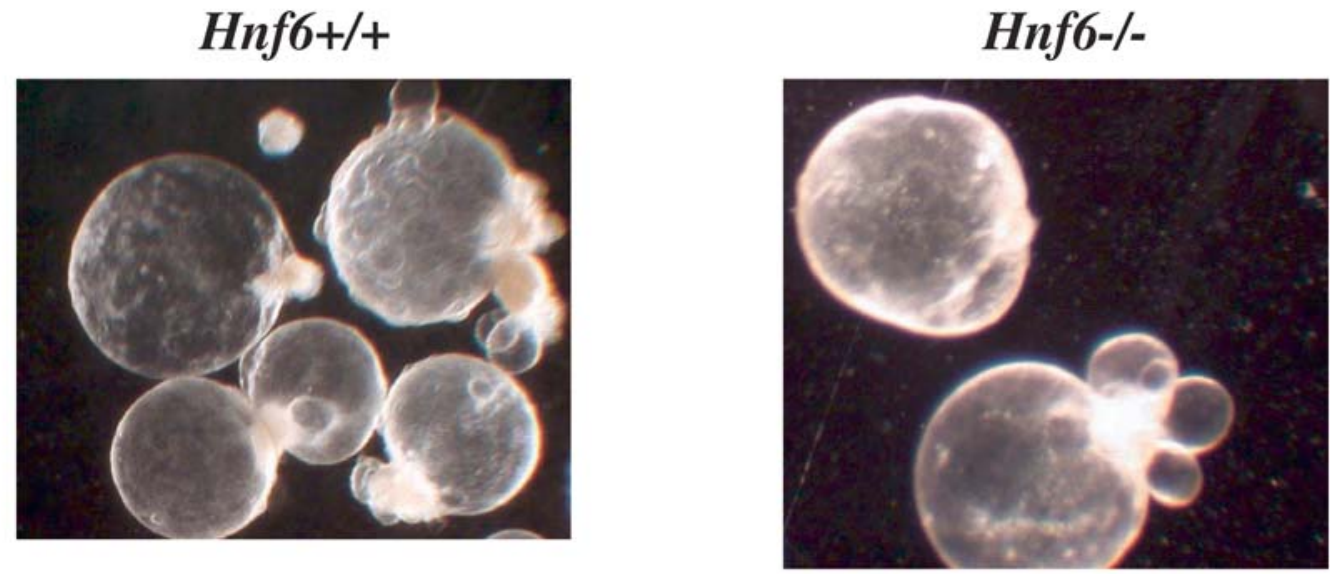

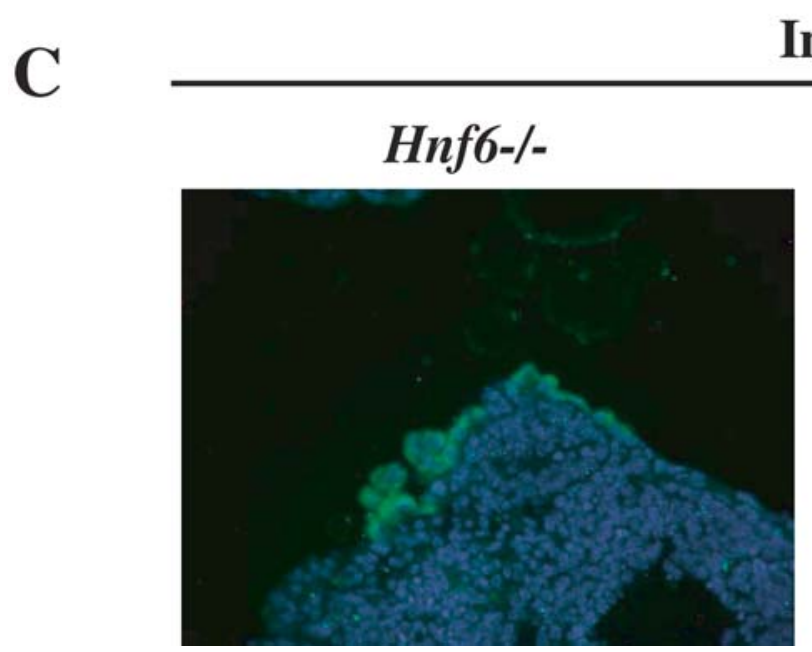

Fig. 3A-C. Detection of insulin-producing cells in Hnf6-/embryoid bodies. A Southern blot (left panel) on ES cell genomic DNA showing the wild-type $(+/+; 12.5 \mathrm{~kb})$ and homozygous mutated $(-/-; 8.5 \mathrm{~kb})$ Hnf6 gene fragments detected with a probe as in [19]. PCR (right panel) of ES cell DNA with primers amplifying the neo gene and exon 1 of the Hnf6 gene confirmed that the two alleles were mutated in the homozygous mutant clone. B Morphology of EBs at day 21 of differentiation. Wild-type $(H n f 6+/+)$ and $H n f 6-/-$ EBs showed the same spherical morphology and cystic cavities (original magnification was x10). C Immunofluorescence. Sections of Hnf6-/- EBs at day 21 of differentiation were subjected to immunofluorescence and analyzed for insulin expression as in Fig. 1A. The original magnifications were $\mathrm{x} 40$ (right panel) and $\mathrm{x} 20$ (left panel). Insulin staining was cytoplasmic
Insulin

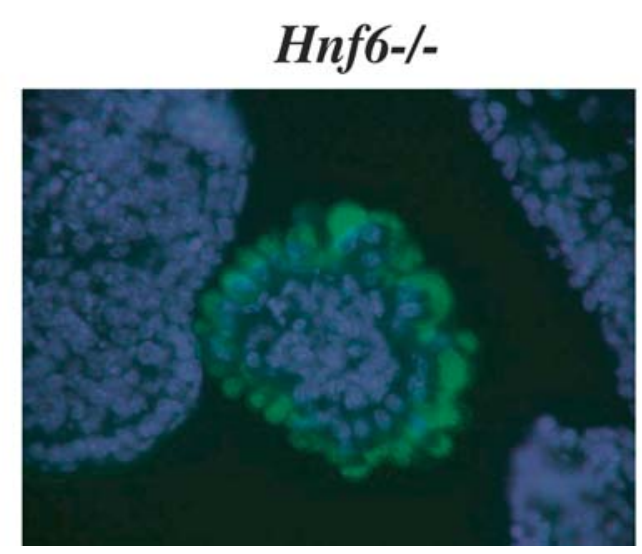

and RNA was collected for analysis by RT-PCR. In contrast to the data obtained in the absence of nicotinamide, the insulin mRNA content increased from day 14 to day 21 with nicotinamide (Fig. 2B). These results demonstrated that the expression of insulin started early and spontaneously during EB differentiation and that it could be stimulated by nicotinamide.

Expression of HNF-6 in embryoid bodies. To examine if HNF-6 was expressed in EBs, we collected the EBs at days 7 and 21 of the suspension culture and analyzed them by immunofluorescence (Fig. 1B). On day 7 , HNF-6 was detected in the nucleus of cells of the outer EB cell layer, while on day 21 HNF-6 was found 
in cells throughout the EBs and no longer in the peripheral cells. A time-course analysis of HNF-6 expression carried out by RT-PCR showed that undifferentiated ES cell expressed HNF-6 (Fig. 2A). This expression transiently increased during the first day of culture in suspension, but returned to lower values soon afterwards. Starting on day 14, the expression of HNF-6 increased again and remained the same until day 21. These observations showed that HNF-6 had a dynamic expression profile during the differentiation of the EBs.

Detection of insulin-producing cells in Hnf6-/- embryoid bodies. To test the requirement of HNF-6 for the differentiation in insulin-producing cells within EBs, we generated HNF-6 null ES cells. Using homologous recombination we had previously obtained $H n f 6+/-$ ES cells in which the first exon of the Hnf6 gene is replaced by a neomycin-resistance cassette. To select Hnf6-/- ES cells we grew the Hnf6+/- ES cells in medium containing different concentrations of G418. Twenty-two ES cell clones survived 9 days in culture with $3 \mathrm{mg} / \mathrm{ml} \mathrm{G} 418$ and were assayed by Southern blotting and by PCR for the loss of the wild-type Hnf6 allele (Fig. 3A). The results indicated that only one clone had lost the wild-type allele. PCR analysis confirmed that this clone consisted exclusively of $H n f 6-/-$ cells.

We then grew the Hnf6-/- ES cells in suspension and in the absence of LIF to allow formation of EBs. The morphology of Hnf6-/- EBs was indistinguishable from that of the wild-type EBs. Their growth was similar and they formed cystic structures like wildtype EBs. To test if the Hnf6-/-EBs produced insulin, we collected the EBs after 21 days of suspension culture and analyzed them by immunofluorescence (Fig. 3C). The results showed that insulin was detectable in a subset of EBs. Like in wild-type EBs, insulin was found in the peripheral cells of the EBs and in some EBs all the peripheral cells expressed insulin. Taken together, the results on insulin expression were identical in wild-type and Hnf6-/- EBs.

To verify if HNF-6 controls the differentiation pattern of insulin-producing cells in the EBs, we compared the time-course of insulin production in wildtype and Hnf6-/- EBs, with or without nicotinamide. In parallel, we also looked at the expression of PDX-1 and NGN-3, two transcription factors that are required for beta-cell differentiation and which are direct targets of HNF-6. We also monitored the expression of HNF-1 $1 \beta$, which is also a direct target of HNF-6 and which, when mutated, is associated with maturity-onset diabetes of the young. Likewise, the expression of the beta cell glucose transporter GLUT-2, another target of HNF-6, was also examined. The results showed that neither the time-course nor the expression of insulin was affected by the absence of HNF-6 (Fig. 2). The slight difference in insulin expression seen between the wild-type and Hnf6-/- EBs was within the range of experimental variations. Similarly, no difference was seen between wild-type and Hnf6-/- EBs in the expression profile of Ngn3, Pdxl, Glut2 and Hnflb (Fig. 2).

In summary, HNF-6 was not required for expression of insulin during differentiation of EBs. Also, none of the genes targeted by HNF-6 in the pancreas during embryonic development showed abnormal expression in the Hnf6-/- EBs. We therefore concluded that HNF-6 is not required for differentiation of insulin-producing cells in EBs.

\section{Discussion}

ES cells can form EBs in which cells spontaneously differentiate into insulin-producing cells. These cells could be used for the therapy of diabetes mellitus. To improve the efficiency with which insulin-producing cells differentiate within EBs, several teams are trying to transpose the beta-cell differentiation mechanisms identified in embryos to the differentiation of EBs. The successful application of this principle requires a knowledge of the spontaneous differentiation mechanisms in the EBs. To approach this question we verified if the differentiation of cells in the EBs mimics that of beta cells in embryos. To do this we tested if the differentiation of insulin-producing cells in EBs requires HNF-6, a transcription factor known to control the differentiation of beta cells in the embryo.

We have shown that insulin is produced in the outer cell layer of the EBs. In EBs this cell layer displays properties that are similar to those of the visceral endoderm, an extraembryonic tissue that participates to several embryonic developmental events and to yolk sac formation $[11,26]$. Indeed, in animals, the visceral endoderm produces a number of proteins such as, for instance, alpha foetoprotein, transthyretin, apolipoproteins and glucose transporters which are also found in the outer cell layer of the EBs [27]. In addition, tissuespecific transcriptional regulators of these genes in the visceral endoderm are functional in the outer layer of EBs $[28,29]$. Insulin is also expressed in the yolk sac visceral endoderm [30], which is consistent with our present observation that insulin is produced by the outer cell layer of EBs. Recent reports indicated that insulin could be produced in neurectodermal tumours [31] and in cells derived from nestin-positive precursors [7, 32, 33], suggesting that insulin can be expressed in cells originating from neuronal precursors. Since cells derived from the primitive ectoderm tend to be localized in the core of the EBs [26], it is unlikely that the insulin-expressing cells found in the outer layer of the EBs derive from neuronal precursors. We therefore suggest that the EB cells which produce insulin behave like visceral endoderm cells.

HNF-6 was found to be expressed in undifferentiated ES cells. This is somehow in contrast with the fact 
that in developing embryos, HNF-6 is expressed in a lineage-restricted manner [17]. However, several lineage-restricted factors, which include HNF-1 $\alpha$, HNF$1 \beta$ and PDX-1 [29, 34, 35], are known to be expressed in undifferentiated ES cells and we show here that this is also the case for NGN-3. More importantly, we found that HNF-6 is also expressed in the outer layer of the EBs, which is consistent with the known expression of HNF-6 in the yolk sac [36]. The expression of HNF-6 in the outer layer of the EBs was transient since at late stages of EB differentiation no HNF-6 was found in these cells. During pancreas development, HNF-6 is found in the endocrine precursor cells [19]. Our study does not rule out that expression of HNF-6 in EBs occurs in precursors of the insulinproducing cells. Indeed, during EB differentiation HNF-6 was detected earlier than insulin. However, in contrast to the requirement of HNF-6 in differentiation of beta cells in embryos, the lack of HNF-6 in EBs had no influence on insulin expression. In embryos, the transcription factors PDX-1 and NGN-3 control pancreas development and endocrine differentiation $[20,37,38]$ and their expression is stimulated by HNF-6 (our unpublished data and reference [19]). In embryos HNF-1 $\beta$ and GLUT-2 are also direct targets of HNF-6 [39, 40] and, when mutated, HNF-1 $\beta$ is associated with maturity-onset diabetes of the young [41]. In EBs the expression of PDX-1, NGN-3, GLUT- 2 and HNF-1 $\beta$ was not affected by the lack of HNF-6.

In summary, none of the known roles of HNF-6 in embryonic beta cell differentiation was recapitulated in the EBs. We conclude that the molecular mechanisms of the differentiation of insulin-producing cells in EBs differ from those of the beta cells in embryos.

Acknowledgements. We thank P. Chomez, O. De Backer, S. Cereghini and the members of our laboratory for help and discussion. The ES cell line R1 was a gift from A. Nagy. N.H. held a PhD fellowship from Télévie (Belgium). This work was supported by grants from the Belgian State Program on Interuniversity Poles of Attraction, (Prime Minister's Office, Federal Office for Scientific, Technical and Cultural Affairs), from the D.G. Higher Education and Scientific Research of the French Community of Belgium, and from the Fund for Scientific Medical Research (Belgium).

\section{References}

1. Efrat S (1999) Genetically engineered pancreatic beta-cell lines for cell therapy of diabetes. Ann NY Acad Sci 875:286-293

2. Soria B, Skoudy A, Martin F (2001) From stem cells to beta cells: new strategies in cell therapy of diabetes mellitus. Diabetologia 44:407-415

3. Ramiya VK, Maraist M, Arfors KE, Schatz DA, Peck AB, Cornelius JG (2000) Reversal of insulin-dependent diabetes using islets generated in vitro from pancreatic stem cells. Nat Med 6:278-282
4. Bonner-Weir S, Taneja M, Weir GC et al. (2000) In vitro cultivation of human islets from expanded ductal tissue. Proc Natl Acad Sci USA 97:7999-8004

5. Cheung AT, Dayanandan B, Lewis JT et al. (2000) Glucose-dependent insulin release from genetically engineered K cells. Science 290:1959-1962

6. Soria B, Roche E, Berna G, Leon-Quinto T, Reig JA, Martin F (2000) Insulin-secreting cells derived from embryonic stem cells normalize glycemia in streptozotocin-induced diabetic mice. Diabetes 49:157-162

7. Lumelsky N, Blondel O, Laeng P, Velasco I, Ravin R, McKay R (2001) Differentiation of embryonic stem cells to insulin-secreting structures similar to pancreatic islets. Science 292:1389-1394

8. Assady S, Maor G, Amit M, Itskovitz-Eldor J, Skorecki KL, Tzukerman M (2001) Insulin production by human embryonic stem cells. Diabetes 50:1691-1697

9. Soria B (2001) In vitro differentiation of pancreatic betacells. Differentiation 68:205-219

10. Weiss MJ, Orkin SH (1996) In vitro differentiation of murine embryonic stem cells. New approaches to old problems. J Clin Invest 97:591-595

11. Doetschman TC, Eistetter H, Katz M, Schmidt W, Kemler $\mathrm{R}$ (1985) The in vitro development of blastocyst-derived embryonic stem cell lines: formation of visceral yolk sac, blood islands and myocardium. J Embryol Exp Morph 87: 27-45

12. Odorico JS, Kaufman DS, Thomson JA (2001) Multilineage differentiation from human embryonic stem cell lines. Stem Cells 19:193-204

13. Kim SK, Hebrok M (2001) Intercellular signals regulating pancreas development and function. Genes Dev 15:111127

14. Edlund H (1998) Transcribing pancreas. Diabetes 47: 1817-1823

15. Lemaigre FP, Durviaux SM, Truong O, Lannoy VJ, Hsuan JJ, Rousseau GG (1996) Hepatocyte nuclear factor-6, a transcription factor that contains a novel type of homeodomain and a single cut domain. Proc Natl Acad Sci USA 93:9460-9464

16. Lannoy VJ, Bürglin TR, Rousseau GG, Lemaigre FP (1998) Isoforms of HNF-6 differ in DNA-binding properties, contain a bifunctional homeodomain and define the new ONECUT class of homeodomain proteins. J Biol Chem 273:13552-13562

17. Landry C, Clotman F, Hioki T et al. (1997) HNF-6 is expressed in endoderm derivatives and nervous system of the mouse embryo and participates to the cross-regulatory network of liver-enriched transcription factors. Dev Biol 192:247-257

18. Rausa F, Samadani U, Ye H et al. (1997) The cut-homeodomain transcriptional activator HNF-6 is coexpressed with its target gene HNF-3b in the developing murine liver and pancreas. Dev Biol 192:228-246

19. Jacquemin P, Durviaux SM, Jensen J et al. (2000) Transcription factor hepatocyte nuclear factor 6 regulates pancreatic endocrine cell differentiation and controls expression of the proendocrine gene ngn3. Mol Cell Biol 20: 4445-4454

20. Gradwohl G, Dierich A, LeMeur M, Guillemot F (2000) Neurogenin 3 is required for the development of the four endocrine cell lineages of the pancreas. Proc Natl Acad Sci USA 97:1607-1611

21. Gu G, Dubauskaite J, Melton DA (2002) Direct evidence for the pancreatic lineage: NGN3+ cells are islet progenitors and are distinct from duct progenitors. Development 129:2447-2457 
22. Apelqvist A, Li H, Sommer L et al. (1999) Notch signalling controls pancreatic cell differentiation. Nature 400: $877-881$

23. Robertson EJ (1987) Embryo-derived stem cell lines. In: Robertson EJ (eds) Teratocarcinomas and embryonic stem cells: a practical approach. IRL press, Oxford, pp 71-112

24. Otonkoski T, Beattie GM, Mally MI, Ricordi C, Hayek A (1993) Nicotinamide is a potent inducer of endocrine differentiation in cultured human fetal pancreatic cells. J Clin Invest 92:1459-1466

25. Mortensen RM, Conner DA, Chao S, Geisterfer-Lowrance AAT, Seidman JG (1992) Production of homozygous mutant ES cells with a single targeting construct. Mol Cell Biol 12:2391-2395

26. Shen MM, Leder P (1992) Leukemia inhibitory factor is expressed by the preimplantation uterus and selectively blocks primitive ectoderm formation in vitro. Proc Natl Acad Sci USA 89:8240-8244

27. Duncan SA, Nagy A, Chan W (1997) Murine gastrulation requires $\mathrm{HNF}-4$ regulated gene expression in the visceral endoderm: tetraploid rescue of Hnf-4 -/- embryos. Development 124:279-287

28. Duncan SA, Navas MA, Dufort D, Rossant J, Stoffel M (1998) Regulation of a transcription factor network required for differentiation and metabolism. Science 281:692-695

29. Abe K, Niwa H, Iwase K et al. (1996) Endoderm-specific gene expression in embryonic stem cells differentiated to embryoid bodies. Exp Cell Res 229:27-34

30. McGrath KE, Palis J (1997) Expression of homeobox genes, including an insulin promoting factor, in the murine yolk sac at the time of hematopoietic initiation. Mol Reprod Dev 48:145-153

31. Nakamura T, Kishi A, Nishio Y et al. (2001) Insulin production in a neuroectodermal tumor that expresses islet factor-1, but not pancreatic-duodenal homeobox 1. J Clin Endocrinol Metab 86:1795-1800
32. Hunziker E, Stein M (2000) Nestin-expressing cells in the pancreatic islets of Langerhans. Biochem Biophys Res Commun 271:116-119

33. Zulewski H, Abraham EJ, Gerlach MJ et al. (2001) Multipotential nestin-positive stem cells isolated from adult pancreatic islets differentiate ex vivo into pancreatic endocrine, exocrine, and hepatic phenotypes. Diabetes 50:521533

34. Schuldiner M, Yanuka O, Itskovitz-Eldor J, Melton DA, Benvenisty N (2000) Effects of eight growth factors on the differentiation of cells derived from human embryonic stem cells. Proc Natl Acad Sci USA 97:11307-11312

35. Gerrish K, Gannon M, Shih D et al. (2000) Pancreatic beta cell-specific transcription of the pdx-1 gene. The role of conserved upstream control regions and their hepatic nuclear factor 3beta sites. J Biol Chem 275:3485-3492

36. Keng VW, Yagi H, Ikawa M et al. (2000) Homeobox gene hex is essential for onset of mouse embryonic liver development and differentiation of the monocyte lineage. Biochem Biophys Res Commun 276:1155-1161

37. McKinnon CM, Docherty K (2001) Pancreatic duodenal homeobox-1, PDX-1, a major regulator of beta cell identity and function. Diabetologia 44:1203-1214

38. Stoffers DA, Thomas MK, Habener JF (1997) Homeodomain protein IDX-1. A master regulator of pancreas development and insulin gene expression. Trends Endocrinol Metab 8:145-151

39. Clotman F, Lannoy VJ, Reber M et al. (2002) The onecut transcription factor HNF6 is required for normal development of the biliary tract. Development 129:1819-1828

40. Tan Y, Adami G, Costa RH (2002) Maintaining HNF6 expression prevents AdHNF3beta-mediated decrease in hepatic levels of glut-2 and glycogen. Hepatology 35:790798

41. Horikawa Y, Iwasaki N, Hara M et al. (1997) Mutation in hepatocyte nuclear factor-1 beta gene (TCF2) associated with MODY. Nat Genet 17:384-385 